\title{
Distributed Control Architecture for Wireless Sensor Networks Using IEC 61499 Function Blocks for Industrial Automation
}

\author{
Reza Abrishambaf, Member, IACSIT, Mert Bal, and Majid Hashemipour
}

\begin{abstract}
Wireless Sensor Networks (WSN) technology is rapidly becoming a feasible solution for monitoring and control at the lowest level of manufacturing automation systems. This paper presents a distributed approach for modeling WSN for applications of intelligent manufacturing automation and control. The proposed approach uses IEC 61499 function blocks, which is a new industry standard in the context of manufacturing automation. The distributed modeling of WSN through the function blocks provides flexibility and scalability to the low-level monitoring and control of the manufacturing systems, which are two important challenges in today's conventional manufacturing control systems. The proposed model is a replacement of the conventional centralized control architecture, where local processing is performed rather than having a central control unit.
\end{abstract}

Index Terms-IEC 61499 Function Blocks, Intelligent Manufacturing, Wireless Sensor Networks.

\section{INTRODUCTION}

The Wireless Sensor Network (WSN) is an emerging technology for a wide range of applications in home automation, habitat and environment monitoring, defense, manufacturing, logistics and many industrial applications. WSN is typically formed by many sensor nodes, having sensing, on-board processing and wireless communication capabilities. Wireless sensors have been effective in monitoring various physical characteristics of the world, including temperature, pressure, flow rate, humidity, acoustics, light and pollution [1]. Due to the rapidly growing sensing and positioning technologies, the WSN has proven its potential to be a viable solution for various low level real-time monitoring and control applications i.e. power plants, residential buildings, warehouses and manufacturing plants [2].

A node in WSN is typically an inexpensive embedded platform, which is capable of low power and multifunctional operations. Each node platform contains several sensors, a microcontroller (MCU), Analog to Digital Converter (ADC)

Manuscript received February 10, 2011; revised July 8, 2011.

Reza Abrishambaf is with the Automation Research Group of the Department of Electrical and Electronic Engineering, Eastern Mediterranean University, Famagusta, Turkish Republic of Northern Cyprus (phone: 0090-392-630-2765; fax: 0090-392-365-0240; e-mail: reza.abrishambaf@ emu. edu.tr).

Mert Bal is with the Department of Engineering Technology, Miami University, Hamilton, OH, USA.

Majid Hashemipour is with the Department of Mechanical Engineering Eastern Mediterranean University, Famagusta, Turkish Republic of Northern Cyprus. and a radio transceiver for communication purposes. With the aid of intelligent and collaborative algorithms, a node may be able to interact with the other nodes in order to identify its neighborhood and establishing a negotiation among them.

Sensors have been one of the most important components for monitoring and control of automated assembly lines and computer integrated manufacturing systems. Various sensors are used to collect real-time data from the manufacturing operations such as; physical shop floor conditions, machine state, location and process status of parts and material handling devices. The information provided by sensors is then processed by the Programmable Logic Controllers (PLCs) in order to control the manufacturing processes. Generally, this type of conventional manufacturing lines cope with two major challenges: i) the real-time data collection in the manufacturing or assembly plant is managed by wired sensors, which are very costly for deployment and maintenance, and lacking the reliability and flexibility that a manufacturing control system typically requires. ii) real-time information is manipulated and processed by centralized controllers. The centralized information processing makes the overall system unreliable and very prone to failures.

In contrast to centralized control systems, the decentralized monitoring and control provides overall system reliable and faster data processing is led to faster decision making [3]. In the decentralized systems, sensing and processing is performed locally in a distributed fashion. Due to its distributed nature, the WSN has a high potential to be the enabling technology of distributed sensing and processing for manufacturing systems, which allow real-time process monitoring, machine diagnostics, condition based monitoring and product quality monitoring [4]. However, the new WSN technology is still so complicated to be implemented into the distributed control of manufacturing systems due to the lack of structured modeling methods and effective guidelines, developed for WSN.

The Multi-agent System (MAS) scheme has been widely utilized in many applications and become very popular in the area of Distributed Control Systems (DCS) for industrial automation and manufacturing. DCS model is a replacement of conventional centralized systems. Instead of utilizing a central control unit, the overall control system will be modeled using local processors such as agents or holons. The agents have authority to be autonomous, cooperative and self organizing.

Several researches have worked on implementing MAS on WSN [5, 6, and 7]. The MAS technology promises a 
considerable potential for modeling WSN for manufacturing applications, since each node may be modeled as an autonomous agent, which is collaborative and even self organizing. However, there is a lack of low level (hardware and embedded system) implementation of the agents on WSN based on a well-known standard.

This paper presents the first phase of our project in Automation Research Group of Eastern Mediterranean University and further results such as simulation and implementation are expected to be appeared in the literatures in near future. The main objective of this paper is to define a generic approach for modeling the WSNs for applications in distributed monitoring and control of manufacturing systems. For this purpose, we introduce a modeling approach for WSNs based on IEC 61499 Function Blocks (FB), which is a new industry standard proposed by International Electrotechnical Commission (IEC) [8] in order to model the distributed control systems. According to the IEC proposal, function blocks are suitable for distributed control and measurements of industrial processes.

The FBs provide scalability and real-time reconfigurability to the manufacturing control system. The real-time reconfiguration is the ability of a system to adapt itself to the dynamic changes, while the system is in operation. It plays an essential role in distributed control systems since it increases the integration among the subsystems, hence improving the efficiency of resources and reducing the number of active components, costs and managing the complexity [9].

The remainder of this paper is as follows; the WSN technology in manufacturing is discussed in Section 2. An overview of IEC 61499 function blocks architecture and the proposed modeling approach are presented in sections 3 . The section 4 discusses the expected behavior of the proposed approach and benefits to manufacturing systems. Finally, the section 5 presents conclusions and identifies the research bearing for future studies.

\section{WIRELESS SENSOR NETWORKS IN MANUFACTURING}

WSNs are newly adopted in the manufacturing area. Wired sensors are subject to sense physical characteristics and send unprocessed data into the central control unit in order to analysis and make decision accordingly. This procedure is prone to time consuming since large amount of data are received by central control unit. Therefore in a situation in which swiftness is vital, this structure is failed. However, in wireless sensor networks, nodes have an on-board DSP processor and therefore by sensing physical characteristics, data processing is performed afterwards and processed data are sent instead. Furthermore, nodes have the ability to collaborate among themselves. This local processing and hence decision making, reduces the response time in a critical situation.

WSNs can be used in different partition of a manufacturing system such as shop floor in order to monitor and control the machines, robots, AGVs, conveyors and etc. Example of smart sensors in a flow-line manufacturing system is given in [3]. Another application of smart sensors in manufacturing equipment and process monitoring and diagnostics, condition based monitoring and product quality monitoring is studied in $[4,11]$. A distributed framework based on smart sensors for machine tools and monitoring is presented in $[12,13]$. Therefore the idea of using WSNs in industrial application is justified in the literatures; however the implementation is not addressed for modern manufacturing systems.

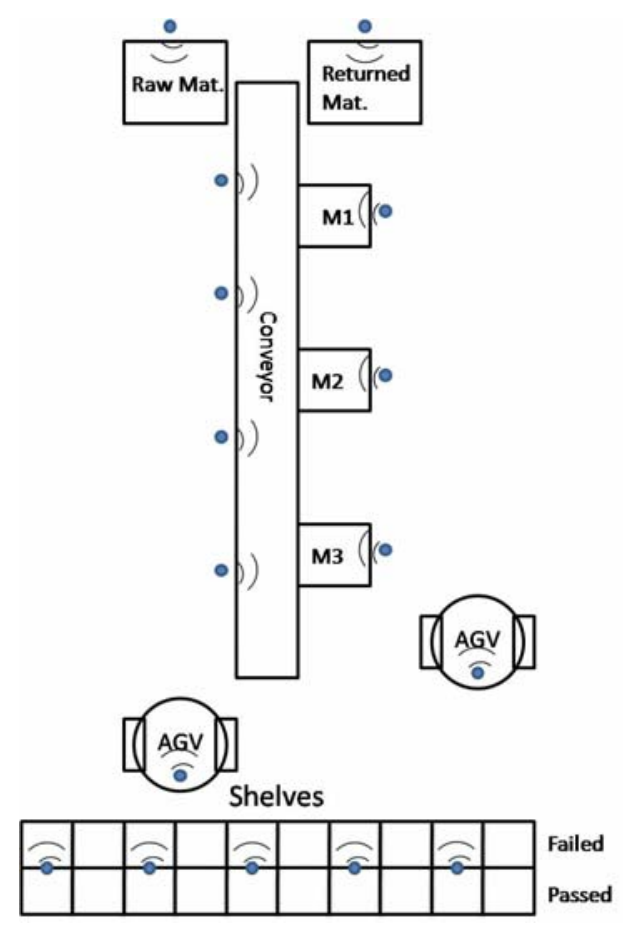

Fig. 1. An Example Assembly Line

Fig 1 is an example of a typical assembly line. Raw materials enter to the line and are carried by means of conveyor. Operations are performed by Machine 1 (M1) and Machine 2 (M2). Machine 3 is supposed to control the part whether the requirements are met. Based on the decision made by M3, the AGV carries the part and puts it to the corresponding shelf. Another AGV is responsible to carry the faulty parts to the return material section for further operation. This assembly line is based on smart sensors which are demonstrated by the dots in Fig 1. Each machine is equipped with a node in order to monitor the status of the machine. As mentioned before, one of the applications of WSN in manufacturing is to monitor the status of machines. Negotiation between the machines in the conveyor could make the system reliable and upon any failure in any machine, the other devices are informed. This will increase the robustness of the system. Flexibility is also achieved since the (re) configuration in any node will be within the framework of IEC 61499 function blocks, which will be explained in the next section.

\section{Proposed Control ARChiteCtURE}

WSNs are decentralized models since it is composed of a large number of intelligent nodes. Nodes have the capability of self-controlling, self organizing and furthermore they have the authority to establish cooperation among themselves. This cooperation is led to achieve the overall system goal whereas in the conventional centralized systems, raw data are collected and forwarded to the central processor in order to fulfill the system requirements. IEC 61499 function blocks is 
one of the standards which turns out to be very popular in the context of distributed control systems.

\section{A. IEC 61499 Function Blocks}

IEC 61499 consists of IEC 61131-3 and IEC 61804 standard. Distributibility and programmability are inherited from these standards and agility is the common property which is required for the dynamic reconfiguration. Physical reconfiguration requires distributed automation and this standard is suitable for the distributed application. Event and data interfaces, software encapsulation and reuse, event driven and state machines, service interfaces, management service and software portability are the characteristics of IEC 61499 [14].

One of the most important properties of the function block is real-time reconfigurability, in which the system can be reconfigured while ensuring to achieve the overall system goal.

According to [14], a distributed system based on IEC 61499 is a system where the devices have communication links among themselves to perform one or more application. Each application comprises one or more instances. In fact, these instances are the function blocks which have communication link among their event and data interfaces.

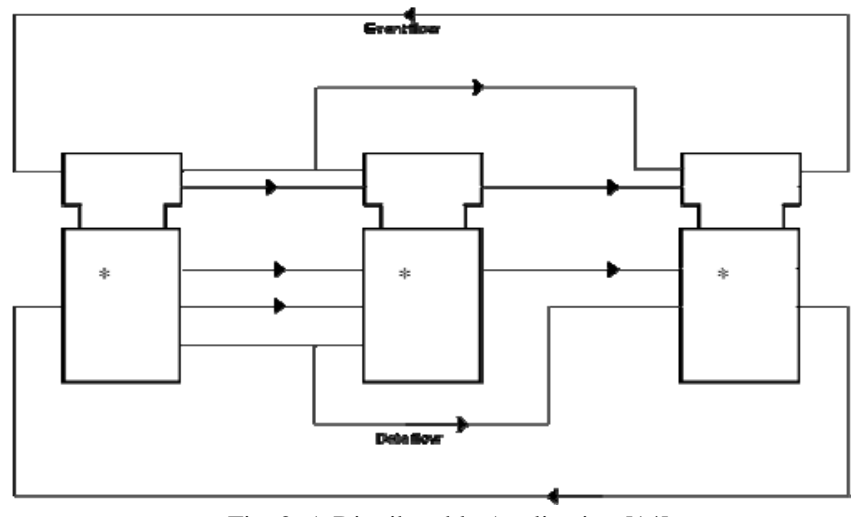

Fig. 2. A Distributable Application [14]

As can be seen from Fig 2, each function block in an application are connected through event and data part. A function block comprises a head and a body. The head is responsible for event inputs and outputs. These events are utilized in order to establish a negotiation with the other function blocks. Execution Control Chart (ECC) is also located in the head part. The control of FB algorithm, time scheduling, event flow control and data flow will be performed by ECC. The body of FB consists of the algorithm and internal variables. The input and output data interfaces are also located in the body. (Fig 3).

A system is a set of devices which are connected by communication link. Each device comprises several resources in which communication interface establishes a connection among resources. On the other hand, data communication between resources and hardware platforms are by means of process interface. One or more resources may be used for an application.

\section{B. Control Architecture}

This section presents the control architecture of the overall system. The proposed model has three tires as shown in Fig 5.

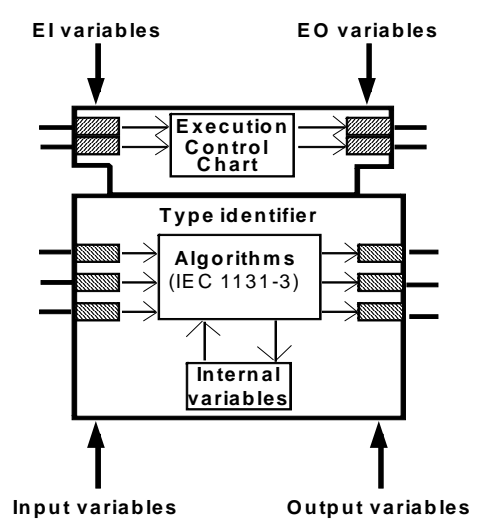

Fig. 3. A Function Block with Event and Data Interface [14]

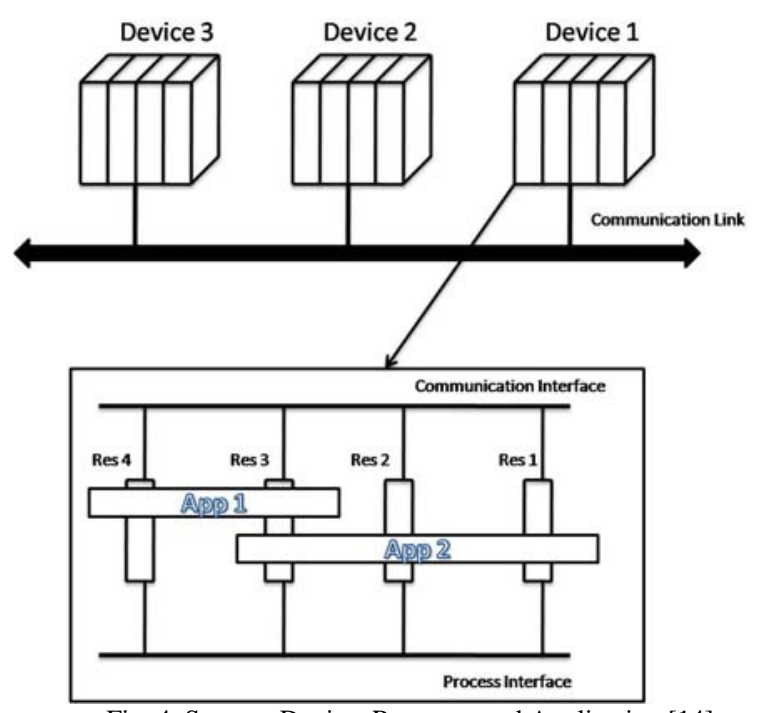

Fig. 4. System, Device, Resource and Application [14]

1) Presentation Tire

This tire is utilized for visualization and graphic user interface in order to establish an interaction among the user and system.

2) Application Tire

Agents, which are located in this tire, are sensor and actuator agents. Normally, this tire is in a distributed fashion so that one or more applications can perform their tasks. This tire is fully compliant with IEC 61499. Real-time reconfiguration and fault monitoring will be performed using the property of IEC 61499 function block. This is a middleware tire and has the main control architecture. It also sends the related data to the presentation tire for visualization.

3) Data Tire

This tire collects the data from the physical devices. This tire is also fully compliant with IEC 61499 function blocks. There are special function blocks call Service Interface Function Blocks (SIFB) which collects data from the hardware tire. Data management and collection are two important factors which are considered in this tire.

4) Sensing and Control Platform

This is the actual physical tire in which sensors and other automation devices are located and connected by means of a network (typically TCP/IP). Interoperability will be achieved through the networked devices (Fig 6).

As can be seen from Fig 6, the application tire is based on 
IEC 61499 standard in a distributed fashion. Agent and Agent platforms, Directory Facilitator (DF) and Agent Management System (AMS) are resided in this tire. Each agent is defined by means of function blocks. The status of each agent, current job and agent ID are in a list so called DF. AMS is responsible for managing the agents' job, new agents, negotiation and other issues. For instance, each sensor node will be defined as an agent in DF and AMS will have full control over the agents or nodes (in this case). In case of failure in one node, a new agent will be created for the new node in.

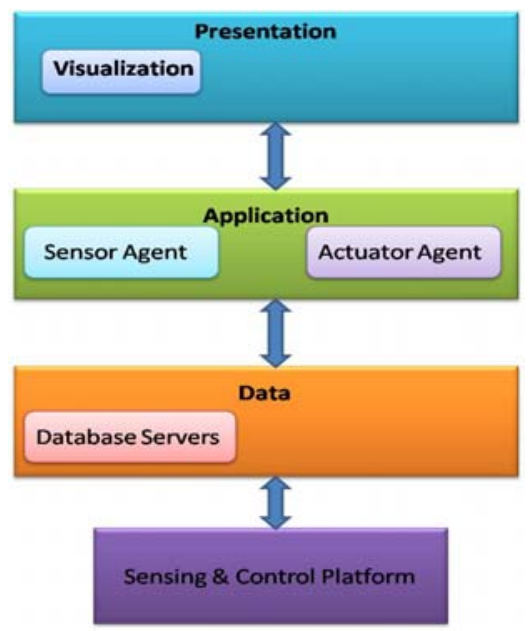

Fig. 5. The proposed control architecture

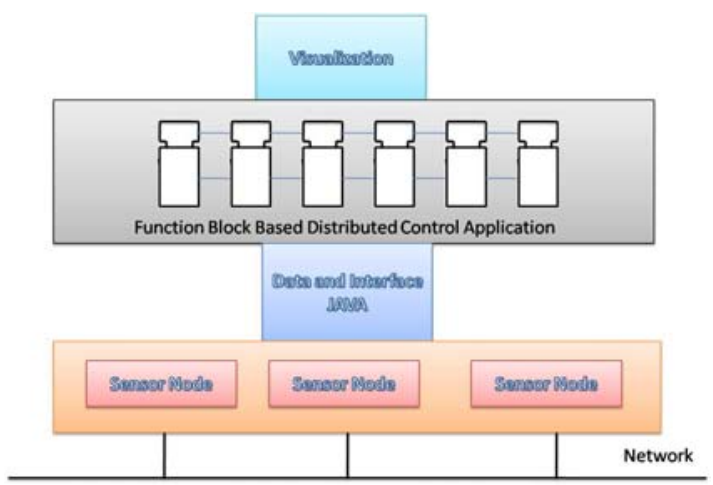

Fig. 6. The proposed control architecture layers

In the proposed model, each resource is considered as a wireless sensor node since it is a functional unit having independent control of its operation. Therefore having a collection of resources or nodes, one or more applications may be implemented. Fig 7 represents the proposed hardware model.

The connection between a node and a resource is established by the dedicated function block called Process Interface Function Block. This FB is responsible for synchronizing the data movement on process interface. In other words, this is for low level or hardware level communication. There is another interface function block called Communication Interface Function Block which is in charge of the connection among the resources and in this case it will be used for node to node communication. The collection of all these function blocks is called Service Interface Function Blocks [15].

The advantages of the proposed model are, first, the WSN is implemented using a well-known and professional standard (IEC 61499 FB). Second, by assigning each node to a resource, one or more application can be performed in a distributed fashion. Third, real-time reconfiguration is one the most important property of IEC 61499 FBs and therefore in case of changes in any level like device, resource and application, reconfiguration will be performed online without disrupting the rest of the system. Online changing is very important in a dynamic system. Depending on the environment in which the system is located, various problems result in malfunctioning one or more sensor. Therefore the ability of the system to adapt its behavior in such a situation and act accordingly is a vital issue. For example, in case of sensor failure, the corresponding resource will be informed and therefore the other resources or nodes will also be informed by means of service interface function blocks and replacing the nodes can be done in real-time. This is the most important property of the proposed model.

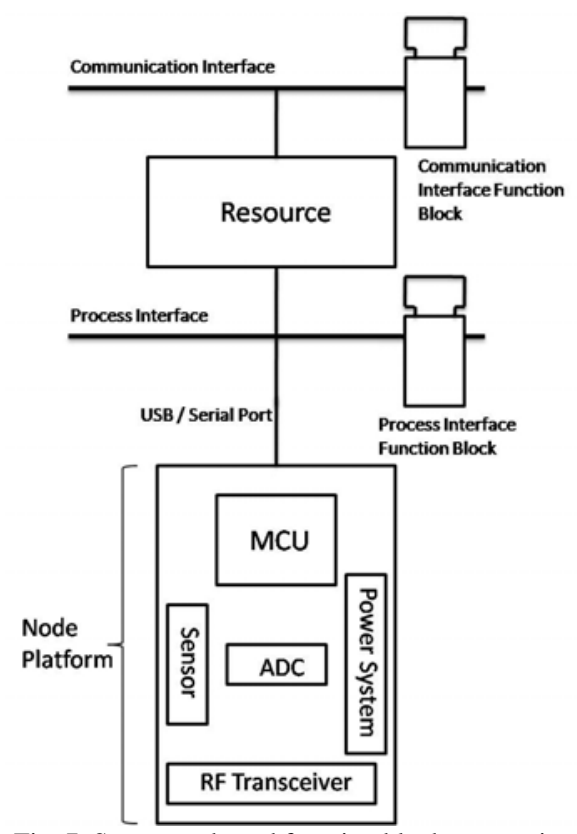

Fig. 7. Sensor node and function block connection

\section{ExPECTED BEHAVIOR OF THE PROPOSED MOdEL}

WSNs are modeled based on IEC 61499 function blocks. This standard has been widely applied in the manufacturing area in order to implement distributed control system. Flexibility and scalability are two important factors which are required in intelligent manufacturing systems. Consider, for instance, an assembly line which is implemented based on IEC 61499 standard, integration of WSNs using this standard to such a system will be straightforward since there will be no need to design different interfaces in order to combine WSNs with system. Furthermore, when integrated, it will increase the flexibility of the system. In case of changes or adding elements, the system will adapt itself faster since the processing will be performed within the function blocks framework (Figure 8).

For instance, consider the engine assembly plant which is proposed by [16]. The system consists of several assembly stations, AGVs, buffer stations and etc. This system was proposed based on holonic manufacturing system. The flexibility and robustness of system is discussed. Consider 
the implementation of such a system based on IEC 61499 function blocks in which WSNs are utilized rather than the wired sensors. Since all the system is based on function blocks, any reconfiguration is within this standard and therefore, no other interfaces are required. This will increase the robustness of the system more comparing with the old fashion.

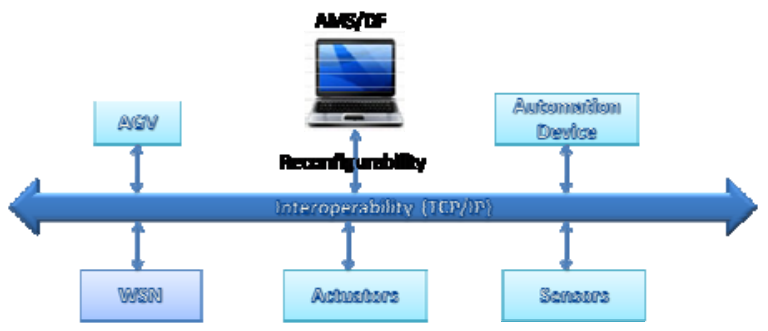

Fig. 8. Integration of WSNs into other Automation Devices

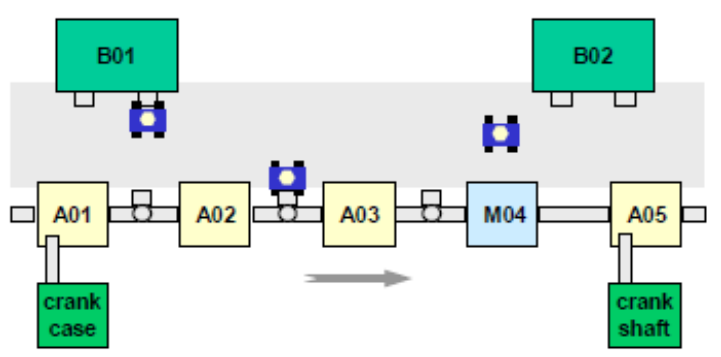

Fig. 9. Engine Assembly Plant [16]

\section{CONCLUSION AND FUTURE WORK}

In this paper, WSNs has been modeled based on IEC 61499 function blocks. This new standard has been adopted widely in manufacturing in order to implement distributed control systems. In the proposed model, these two new technologies are merged intended for increasing flexibility and scalability of the manufacturing systems. The next phase of this project will be the simulation of the model and implementation on a manufacturing system such as small or medium size company.

\section{REFERENCES}

[1] F. Akyildiz, W. Su , Y. Sankarasubramaniam , E. Cayirci, “ Wireless sensor networks: a survey" , Computer Networks: The International Journal of Computer and Telecommunications Networking, v.38 n.4, p.393-422, 2002.

[2] Mert Bal, Min Liu, Weiming Shen, Hamada Ghenniwa, "Localization in Cooperative Wireless Sensor Networks: A Review", Proceedings of 13th International Conference on Computer Supported Cooperative Work in Design, Santiago, Chile, 2009.

[3] Vadd, S. Kamarthi, S.V. Berry, N.M. "Smart sensors in a flow-line manufacturing system: an agent-based simulation”, International
Conference on Integration of Knowledge Intensive Multi-Agent Systems ,pp. 635- 640, 2005.

[4] Vadde. S., Kamarthi, S., V. and Gupta, S. M. "Modeling Smart Sensor Integrated Manufacturing Systems", Proceeding of the SPIE International Conference on Intelligent Manufacturing, Providence, Rhode Island, pp. 30-37, 2003.

[5] Richard Tynan, G.M.P. O'Hare, David Marsh, and Donal O'Kane, "Multi Agent System Architecture for Wireless Sensor Networks", Workshop on "Intelligent Agents in Computing Systems" - The Agent Days in Atlanta, 2005.

[6] Richard Tynan, G.M.P. O’Hare, David Marsh, and Donal O'Kane, "Intelligent agents for wireless sensor networks", Proceedings of the fourth international joint conference on Autonomous agents and multiagent systems, pp. 1179-1180, 2005.

[7] Meritxell Vinyals; Juan A. Rodríguez-Aguilar; Jesús Cerquides, "A Survey on sensor Networks from a Multiagent Perspective", Artificial Inteligence Research Institute, Spanish National Rsearch Council, The Computer Journal (In Press).

[8] International Electrotechnical Commission, "IEC 61499-1: Function Blocks - Part 1 Architecture”, International Standard, First Edition, Geneva, 2005.

[9] Luis Almeida, Paulo Pedreiras, An ARTIST2 Workshop on Networks for Reconfigurable Embedded Systems, NeRES, Aveiro, Portugal, 2007.

[10] M. Bayartm "Smart Devices for Manufacturing Equipment”, Robotica, vol. 21, pp. 325-333. Cambridge University Press, 2003.

[11] Jeffrey J. Evans." Wireless sensor networks in electrical manufacturing ", Proceedings Electrical Insulation Conference and Electrical Manufacturing Expo. pp. 460-465, 2005.

[12] Xavier Desforges Contact Information, Abdallah Habbadi, Laurent Geneste and François Soler, "Distributed machining control and monitoring using smart sensors/actuators", Journal of Intelligent Manufacturing, pp. 39-53, 2004.

[13] Xavier Desforges and Bernard Archimède, "Multi-agent framework based on smart sensors/actuators for machine tools control and monitoring”, Engineering Applications of Artificial Intelligence, vol. 19, issue 6, pp. 641-655, 2006.

[14] James H. Christensen, "Basic Concepts of IEC 61499" Presented at: Distributed Automation 2000, Magdeburg, Germany, 2000.

[15] Valeriy Vyatkin, "IEC 61499 Function Blocks for Embedded and Distributed Control Systems Design" O3 Neidia and Instrumentation Society of America (ISA), 2007.

[16] Stefan BUSSMANN, Jörg SIEVERDING, "Holonic Control of an Engine Assembly Plant an Industrial Evaluation" Proceedings of the IEEE Systems, Man, and Cybernetics Conference, pp. 169 - 174, Tucson. USA, 2001.

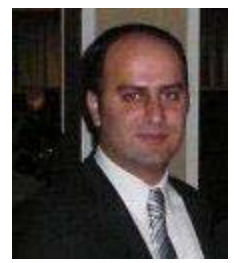

Reza Abrishambaf was born in Shoushtar, Khouzestan, Iran in 1982. He received his MSc degree in Electrical and Electronic Engineering from Eastern Mediterranean University, Northern Cyprus in 2007. He joined the same department for continuing his study as a $\mathrm{PhD}$ student. His research interests include Distributed Control Systems (DCS), Intelligent Manufacturing, Wireless Sensor Networks and IEC 61499 Function Blocks.

He is currently a PhD Candidate and Instructor in Eastern Mediterranean University, Northern Cyprus. He has been working as Research Assistant in the Department of Electrical and Electronic Engineering of Eastern Mediterranean University since February 2006.

Mr. Abrishambaf is an IEEE Gold and Graduate Student Member and also IEEE Circuits and Systems Society, IEEE Robotics and Automation Society and IACSIT. He is a reviewer of several international journals and conferences. 